\title{
Aportes para pensar la integración docencia, investigación y extensión universitaria, desde una experiencia en el campo "envejecimiento y vejez"
}

\section{María del Carmen Ludi \\ mcludi@fibertel.com.ar}

Carina Messina

carinamessina@gmail.com
Docentes investigadoras de la Facultad de Trabajo Social. Universidad

Nacional de Entre Ríos, Argentina.
Integración de la docencia y la extensión /

Intervenciones

RECEPCIÓN: 11/06/16

ACEPTACIÓN FINAL: 23/09/16

\section{Resumen}

El objetivo del artículo es compartir algunas reflexiones desde una perspectiva de integración con relación a nuestra práctica docente en las cátedras Intervención Profesional ${ }^{1} \mathrm{y}$ en proyectos de extensión e investigación en el campo "envejecimiento y vejez".

Se trabajan dimensiones que conforman dicha articulación, las que posibilitan mostrar el potencial de generación de conocimiento e intervención de la universidad en cuanto a la realidad social en que se inserta y de la cual forma parte.

En el desarrollo de la práctica preprofesional de los estudiantes, la extensión universitaria y la investigación, se constituyen en herramientas fundamentales para el trabajo de diferentes temáticas, en nuestro caso "envejecimiento y vejez". Especialmente, teniendo en cuenta la inserción en organizaciones institucionales, público estatales y público societales, con las que se comparte la producción de saberes y diseño de estrategias de intervención tendientes a transformar situaciones problemáticas ligadas a las manifestaciones de la cuestión social.

Palabras-clave

- Integración docencia-extensión-investigación

- Vejez

\section{Resumo}

O objetivo do artigo é compartilhar algumas reflexões a partir de uma perspectiva de integração, em relação à nossa prática docente nas cadeiras: Intervenção Profissional e projetos de extensão e pesquisa no campo "Envelhecimento e velhice".

Dimensões que fazem esse tipo de trabalho conjunta, que tornam possível para mostrar o potencial de geração de conhecimento e envolvimento da universidade em relação à realidade social em que está inserido e do qual faz parte.

No desenvolvimento de uma prática pré-profissional dos estudantes, extensão universitária e de pesquisa constituem ferramentas fundamentais no trabalho de diferentes temas, no nosso caso "Envelhecimento e velhice". Especialmente considerando a inserção em organizações institucionais, estado públicos público e social, com as quais a produção de estratégias de conhecimento e de intervenção de design projetado para transformar situações problemáticas relacionadas com as manifestações da questão social é compartilhada.

Palavras-chave

- Integração docencia-extensão-investigação

- Velhice

\section{Para citación de este artículo}

Ludi, M. C. y Messina, C. (2016). Aportes para pensar la integración docencia, investigación y extensión universitaria, desde una experiencia en el campo envejecimiento y vejez. En Revista +E versión digital, (6), pp. 298-305. Santa Fe, Argentina: Ediciones UNL. 


\section{Introducción}

La articulación entre equipos de proyectos (de extensión e investigación) y cátedras en nuestra Facultad es un espacio muy rico y sostenido en el tiempo, el que consideramos muy importante ya que configura la base de la relación que la universidad construye con la sociedad.

Estas experiencias nos permiten otorgar sentido a dicha relación como bien y espacio público en el marco de un claro proyecto político, académico y pedagógico.

Hoy, una vez más, la universidad está puesta en "tela de juicio", tanto en nuestra sociedad como en la comunidad educativa. Las transformaciones contextuales tallan hondo, algunas han dejado aún huellas inscriptas en tendencias instrumentales, hegemónicas y fortalecidas por sobre las de tipo liberadoras, emancipadoras. De allí el desafío y compromiso de la universidad pública por superarlas y continuar elaborando propuestas innovadoras.

\section{Aspectos y dimensiones que configuran las "funciones básicas" de la universidad}

Para poder recuperar nuestra experiencia, creemos necesario hacer algunas consideraciones conceptuales y reflexivas en torno a aspectos y dimensiones que configuran las funciones acordadas como básicas de la universidad y que constituyen la base de nuestro trabajo.

\subsection{Acerca de la práctica docente}

Preguntarse sobre el ejercicio de la docencia: por qué, para qué, con quiénes, en este contexto, en este país, en esta Universidad, en el marco de las Ciencias Sociales, de tensiones nuevas y otras viejas resignificadas, ha constituido siempre un nudo problemático. Seguramente en muchos momentos nos paraliza el hecho de "enfrentar" a los estudiantes en clase, en talleres, en jornadas de trabajo: qué decirles, qué mensaje expresar, cómo rearmarnos para seguir, cómo hacer para que los idearios no se derrumben, la utopía no muera, la esperanza siga en pie, la primavera no se detenga. Sobre todo en el trabajo docente con las últimas generaciones de jóvenes, ante cambios muy profundos de valores, de lógicas, de sentidos.

Paulo Freire (1997) sostenía que enseñar exige: rigor metódico, investigación; respeto a los saberes y autonomía de los educandos; actitud crítica; estética y ética; testimonio; no discriminación; apertura a lo nuevo; reconocimiento de la identidad cultural; conciencia de lo no acabado; compromiso, seguridad, competencia profesional; no egoísmos; libertad y autonomía; toma de decisiones; saber escuchar; reconocer que la educación tiene una base de sustentación ideológica; disponibilidad para el diálogo; tener como base el respeto, la confianza y el afecto; pasión y esperanza; algunas convicciones acerca de las transformaciones. Cuántas expectativas y requisitos puestos en personas, en sujetos, en "uno". Y allí están el desafío, las posibilidades y las condiciones, ya que no podemos dejar de reconocer que nos interesa estar en "ese" lugar, tener parte de ese poder que brinda el ser docente, aun desde una concepción de relación horizontal, sin perder la asimetría necesaria en la que se juega la cuestión ética en la relación docente-estudiantes y que tiene el papel principal de hacer que el respeto por el pensamiento del otro, de la apertura a lo diferente, la posibilidad de diálogo, no sean sólo una mera "declaración". Práctica docente en el ámbito universitario, en el marco de un proyecto político-pedagógico que surge de la confrontación de distintos actores y grupos de la institución, que se materializa a través de una determinada organización discursiva, en un perfil académico, en líneas de investigación y extensión, en las carreras a dictar, en los diseños curriculares a sostener. Proyecto que muestra una opción de carácter político y no meramente técnico en tanto expresa una concepción de universidad y un tipo de vinculación de ésta con la sociedad; el modo de entender la ciencia, la producción de conocimientos y sus aportes a la sociedad.

En este sentido, recuperamos la idea de "profesores como intelectuales transformativos" que planteara Henry Giroux (1990).

Para el autor, toda actividad humana implica alguna forma crítica teórica de las ideologías tecnocráticas e instrumentales subyacentes a determinadas teorías educativas; los profesores tienen que desempeñar un papel responsable en la configuración de los objetivos y las condiciones de la enseñanza...; si los profesores han de educar a los estudiantes para ser ciudadanos activos y críticos, deberían convertirse ellos mismos en intelectuales transformativos. Para Giroux, el conocimiento y el poder están inextricablemente ligados a la presuposición de que escoger la vida, reconocer la necesidad de mejorar su carácter democrático y cualitativo para todas las personas, equivale a comprender las condiciones previas necesarias para luchar por ello. Hacer lo político más pedagógico significa servirse de pedagogías que traten a los estudiantes como sujetos críticos, hacer problemático el conocimiento, recurrir al diálogo, concederles voz y voto en sus experiencias de aprendizaje; desarrollar un lenguaje propio atento a los problemas experimentados en el nivel de la vida diaria, con experiencias conectadas con la práctica del aula...; el punto de partida son los estudiantes como grupos en sus múltiples contextos culturales, de clase social, raciales, históricos y sexuales,
Trabajo Social, Universidad Nacional de Entre Ríos. 
juntamente con la particularidad de sus diversos problemas, esperanzas y sueños... en definitiva, los profesores como intelectuales transformativos necesitan desarrollar un discurso que conjugue el lenguaje de la crítica con el de la posibilidad de cambio, de transformación.

\subsection{Acerca de la investigación}

En nuestra Facultad, la problematización sobre la relación investigación-intervención ha ocupado un espacio importante en la agenda de discusiones, fortaleciendo la práctica investigativa. También la direccionalidad otorgada al plan de estudios vigente de la Licenciatura en Trabajo Social (2001) reconoce en su base la estrecha relación entre interpretación-intervención, como intento de superar la histórica dicotomía entre pensar/hacer, la que ha mostrado su inconsistencia como fundamento de la intervención profesional. Todo "hacer" surge de un "modo de ver y de nombrar" (Teresa Matus, 1999), de allí que los procesos de intervención, están sustentados en teorías (implícitas o explícitas), lo que implica tensionar las diferentes herramientas teóricas con los procesos sociales en los cuales Trabajo Social interviene. Resulta sumamente necesario, la construcción de mediaciones que posibiliten comprender y dar cuenta de la estructura y dinámica social, a modo de desciframiento de la realidad social (Marilda lamamotto, 2003), que posibiliten delinear propuestas de trabajo creativas, propositivas y capaces de preservar y tornar efectivos los derechos.

Estos "modos de ver" las interpretaciones que realicemos, las propuestas de intervención que generemos, son los que producen sentido, y es en ésta línea que consideramos que la universidad en su conjunto y nosotros como uno de sus principales actores tendríamos que preguntarnos sistemáticamente acerca de nuestras prácticas: docente, de extensión, de investigación. Instancias que generan condiciones para hacer más fructífera la relación enseñanza-aprendizaje. En el ámbito de la universidad estas funciones se plantean con tiempos, ordenamientos, presupuestos y lógicas distintas; separadas a pesar de los intentos de unirlas. De allí que es importante imaginar posibles articulaciones, inscriptas en la idea de que el compromiso docente también requiere provocar la capacidad de pensar sobre cuestiones y preguntas que orientan la misma. De esta manera estaremos en condiciones de hacer mejores aportes a la formación profesional: motivando la actitud investigativa y de apropiación de una perspectiva analítica crítica.

Carmen Lera (2006) plantea que Trabajo Social tiene importantes acumulaciones provenientes de sus intervenciones y la investigación es el recurso que disponen las disciplinas del campo social para comprender y explicar hechos, relaciones, estructuras; en síntesis para "nombrar" e "interpretar" fenómenos y procesos sociales. El "discurso" de Trabajo Social paulatinamente ha comenzado a circular, sabiendo que lo que está en juego es la autoridad científica, la capacidad de imponer criterios de verdad, de cientificidad. No es poca la energía puesta, ya que se trata de un espacio de lucha altamente competitivo y cuyas reglas de juego están ordenadas en base a criterios elaborados desde posiciones dominantes que acreditan y certifican las producciones científicas, motivo aún pendiente de discusión en la universidad argentina. La inquietud por la investigación estuvo presente en la profesión prácticamente desde sus inicios, con distintas consideraciones respecto a su función, a las características, los fines, etc.; fundamentalmente restringida a los ámbitos académicos. En nuestro contexto nunca logró constituirse en una perspectiva asumida mayoritariamente por el colectivo disciplinar, sino que más bien tuvo una ubicación marginal. La producción de conocimientos como expresión de la práctica investigativa, fue caracterizada como manifestación de posturas teoricistas, y por lo tanto desencontradas de la intervención y de los intereses reales y genuinos del Trabajo Social. Una especie de cautela nos ha recorrido alertándonos acerca de los peligros de desviarnos hacia el academicismo, bajo la supuesta idea de alejarnos de los problemas reales que vive la gente. Actitudes que paralizan el pensamiento autónomo, que impiden la imaginación creadora. Según la autora, Trabajo Social debe afrontar una doble responsabilidad disciplinar. Por un lado, al intentar dar cuenta de una porción/región de "lo social" sembrada de contradicciones, de injusticias, de alientos y esperanzas, resignificando y dando contenido a categorías que permitan nombrar lo que acontece. Por otro, al abordar cotidianamente situaciones problemáticas proponiendo alternativas modificadoras de las mismas, pugnando 
para que dichos procesos sociales adquieran visibilidad y sean incorporados a la agenda de discusión pública.

En la misma línea, consideramos que:

"hoy día, no solo Trabajo Social, sino las Ciencias Sociales en su conjunto, deberían aportar desde el conocimiento, a dar cuenta de la configuración actual del mundo social, intentando develar los fenomenales cambios generados en la vida social a partir de las manifestaciones que la cuestión social tiene en la vida cotidiana de las clases sociales subalternas. Para el caso del Trabajo Social, la investigación a su vez, debe servir para problematizar las cuestiones específicas del ejercicio profesional, en tanto ella tiene cercanía con dichas manifestaciones, entendidas como 'problemas sociales'. En este sentido, la investigación no es un requerimiento academicista, es por el contrario un requisito ético-político que nos posiciona frente a la realidad con una conciencia de descubrimiento e interpelación crítica.

No cabe duda que el neoliberalismo devenido en ideología persiste actualmente, lo que trata es de propiciar un pensamiento tecnocrático vinculado sólo a las necesidades del mercado, secundarizando en contraposición, el conocimiento crítico de la vida social...; la práctica y la teoría no son dimensiones separadas, por lo tanto el conocimiento no está separado de la acción, y en ese sentido la existencia de una, implica la existencia de la otra. La práctica como fundamento de la teoría, está vinculada a las necesidades del hombre social y ella es producto de un conjunto de relaciones sociales que aparecen difuminadas, fragmentadas, que es necesario recomponerlas a través del pensamiento y la recreación de conceptos y categorías. La práctica profesional es el campo de expresiones problemáticas que sólo pueden ser analizadas a partir de un conocimiento anterior y que se recrea en el encuentro con la realidad". (Rozas Pagazas y Ludi, 2009)

\subsection{Acerca de la extensión}

La extensión universitaria en sus múltiples sentidos, es un concepto que tradicionalmente ha permitido pensar la relación del 'adentro' con el 'afuera' institucional; posibilita configurar la relación de la Universidad y el contexto en que se inserta, centralmente el para qué de la formación académica, de la producción de conocimientos.

En el Estatuto de la Universidad Nacional de Entre Ríos (UNER) (al igual que en la mayoría de las universidades nacionales) se define que la extensión, la docencia y la investigación constituyen las misiones fundamentales de la misma, pero no hay claridad aún en su conceptualización ni acuerdo en la diversidad de prácticas que pueden incluirse dentro de lo que se considera extensión. Podemos afirmar, compartiendo con Wilhelhy Von Wolf que "el concepto tiene un resabio de categoría residual. Hay una tendencia a usar el concepto de extensión universitaria en la cómoda concepción de todas aquellas actividades 'no clasificables' como docencia e investigación" (citado por Almeida Pastor, 1993).

Cada dispositivo de extensión plantea un proceso de comunicación con diversos actores, sectores y organizaciones, en el que la universidad brinda sus aportes y simultáneamente toma de su medio social, inquietudes, propuestas y posibles resoluciones a diferentes problemas de la comunidad.

En Trabajo Social, se ha considerado históricamente a las prácticas preprofesionales que realizan los estudiantes, como una tarea de extensión universitaria vinculada también a la docencia. En la gran mayoría de las universidades, la extensión constituye en una actividad habitual desarrollada con el propósito de responder a las necesidades de la sociedad, poniéndose casi siempre al servicio de los sectores más vulnerados en sus derechos.

No obstante, en los últimos años y no ajena al contexto de los '90, ha cobrado fuerza otra perspectiva de extensión universitaria que reconoce su tradición en el modelo norteamericano, asociado a la prestación de servicios y la extensión rural: perspectiva economicista e instrumental que en la actualidad se reformula y se redefine como transferencia tecnológica.

Así, las universidades se convierten en agentes de innovación científico tecnológica, asumiendo algunas, un rol de prestador de servicios arancelados, destinados especialmente al sector productivo y privado. La rentabilidad, la eficiencia y el impacto en el medio son criterios que priman en esta modalidad de vinculación, entendiendo a la misma como una fuente alternativa 


\section{6}

los profesores como intelectuales

transformativos necesitan desarrollar

un discurso que conjugue el lenguaje

de la crítica con el de la posibilidad

de cambio, de transformación

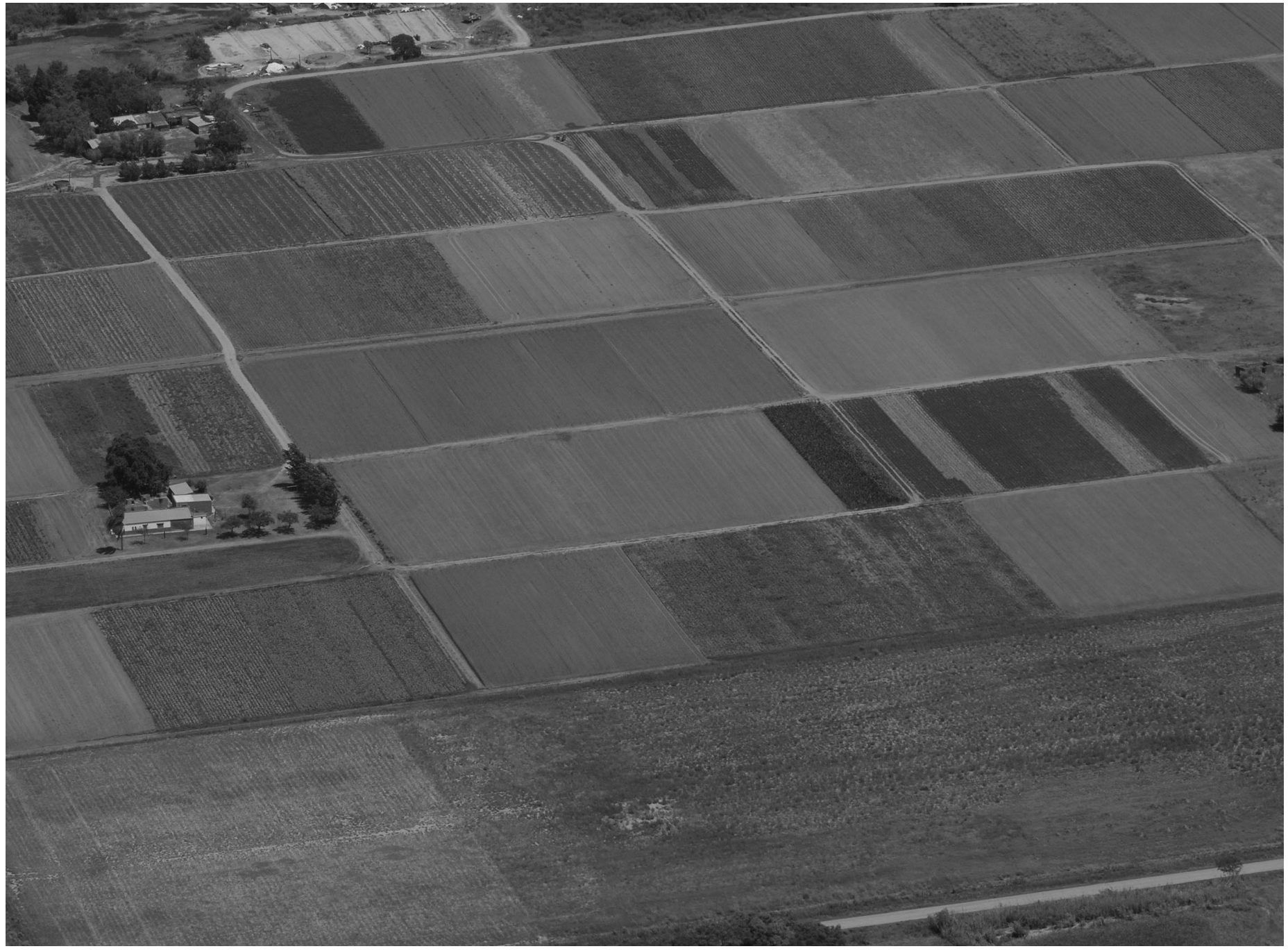

() Hugo Pascucci 
de financiamiento, en su esfuerzo por reestructurarse y compensar la disminución del presupuesto asignado para su funcionamiento; obteniendo recursos complementarios de los sectores privados y hasta del mismo Estado. La postura e insistencia de la Universidad en el autofinanciamiento de la extensión universitaria remite no solo a la consideración de ésta como actividad residual y de baja consideración y reconocimiento — se le otorga un lugar secundario respecto de las otras: no se categoriza, no se remunera- sino también a la puesta en cuestión de proyectos que están dirigidos a sectores sin capacidad de compra de servicios. Se plasma igualmente en los escasos recursos que se destinan para llevar adelante los diferentes proyectos, instando a la voluntad de quienes tienen interés en desarrollar actividades de naturaleza "solidaria", como plus del trabajo docente, constituyéndose como la "hermana pobre" de la investigación y la docencia, salvo en aquellas Facultades en las que la venta de servicios se ha convertido en fuente de ingresos y financiamiento, tal como mencionáramos. Más recientemente, se viene instando a la curricularización de la extensión como modo de relación, intercambio y aportes de la Universidad a través de tareas realizadas por los estudiantes de distintas carreras, máxime en aquellas que aún no contemplaban en su Plan de Estudios, algún tipo de inserción o intervención social. En nuestra Facultad los proyectos de extensión están íntimamente vinculados a cátedras y a proyectos de investigación, lo que posibilita un enriquecimiento permanente entre las distintas tareas que se realizan en la universidad, multiplicando espacios, contribuyendo a la formación de recursos humanos e instalando debates sobre problemáticas generalmente silenciadas, principalmente desde el Estado. Se participa además en el desarrollo de seminarios temáticos (obligatorios u optativos) que contribuyen al acceso de instancias académicas por parte de los estudiantes de la UNER, de otras universidades y de la sociedad. Lo que se pone en juego es la producción de conocimiento sobre la realidad, en conjunto con otros "no universitarios", el hacer visible temas y "problemas".

"Esto implica transitar el difícil camino de 'salir de', en una acción cotidiana, diseñada e implementada a través de la participación de la comunidad educativa en su conjunto: docentes, estudiantes, investigadores, graduados y no docentes, siendo así protagonistas de los principales acontecimientos de la región, junto a organizaciones de la sociedad civil, con el fin de trabajar con éstas sobre los problemas que plantea la misma comunidad. Esta relación entre el conocimiento producido, el capital humano disponible, y los problemas más críticos, constituyen el núcleo duro de la extensión.
Consideramos altamente significativo ir más allá del concepto de acciones de transferencia, es necesario plantearla desde y hacia una construcción dialéctica entre las diferentes instituciones, organizaciones, actores sociales y políticos que conforman la sociedad, como interacción social compleja. Se convierte en un nudo de tensión de la vida académica que propicia un 'punto de provisorio equilibrio' entre la fascinación de la teoría, la discusión académica infinita y la urgencia de conocimiento y acción pertinentes para comprender realidades locales y actuar en la dirección de aportar a la resolución de problemas". (AA. VV., 2007)

\section{Nuestra experiencia de articulación en el campo "envejecimiento y vejez"}

En el marco de la práctica académica pre profesional, se articulan e integran las asignaturas Intervención Profesional y Vida Cotidiana ( $3^{\circ}$ año, 384 horas) e Intervención Profesional e Institucionalidad Social ( $4^{\circ}$ año, 384 horas). ${ }^{2}$ La propuesta se caracteriza por mantener una línea de continuidad e integración en la formación del estudiante, a partir del compromiso asumido con diferentes actores involucrados en el proceso de aprendizaje. A fin de garantizar la modalidad de trabajo, los docentes elaboramos proyectos de trabajo (que incluyen o parten de cátedras, proyectos de extensión y/o investigación, organizaciones institucionales) que favorezcan la permanencia en el tiempo y una perspectiva inter o multidisciplinaria. Se consolidan procesos de intervención, áreas temáticas, espacios de trabajo; intentando construir estrategias que articulen instancias de docencia, extensión e investigación, involucrando a diferentes sectores sociales: organizaciones de la sociedad civil, organizaciones del Estado, organizaciones privadas y movimientos sociales.

El "recorte" temático se convierte en un "pretexto", en facilitador; sirve para referenciar un campo, ligado a las manifestaciones de la cuestión social, a espacios de inserción laboral. Permite trabajar la relación: universal-particular-singular en la Intervención Profesional en tanto campo problemático; problemáticas sociales, priorizándose la preeminencia del aprendizaje de la intervención profesional; del oficio, por sobre la temática.

En nuestro caso, partimos de considerar que "envejecimiento y vejez" se constituye en una de las principales temáticasproblemáticas de este principio de siglo, relacionado principalmente al cambio sustancial en la composición demográfica. Sin dudas, en los últimos años, esto ha tenido y tendrá en las próximas décadas, gran influencia en la prestación de servicios para la población
2) Consultar Programación Académica Anual aprobada por Consejo Directivo de la Facultad de Trabajo Social.
Página web FTS/UNER. Secretaría Académica / Programas de Cátedra 
anciana, dado el incremento considerable en cuanto a necesidades; requerimientos: físicos; afectivo-psicológicos y socioculturales. Sus consecuencias son múltiples tanto para los propios viejos, sus familias, como para la sociedad en general, ya que el envejecimiento de la población constituye un factor importante en el sistema económico, político, social y cultural.

Nuestro país, al igual que nuestra provincia, tiene características de "población envejecida" por lo que se hace necesario problematizar esta situación a futuro, por lo que apelamos al rol indelegable que debe cumplir el Estado, a nivel nacional, provincial y local, en cuanto a protección social, en la formulación e implementación de políticas sociales.

La vida cotidiana y los procesos de envejecimiento particulares, van configurándose cada vez con mayores "dificultades a vencer", condenando a personas de vastos sectores de nuestra sociedad a envejecer en un contexto de desprotección, de no ejercicio de sus derechos que posibiliten la construcción de ciudadanía plena. Allí reside una de las clave problemáticas para los Trabajadores Sociales del campo de la Vejez: la relación tensional sujetonecesidad en el marco de la cuestión social y que tiene que ver con las posibilidades y modos que tienen viejos y viejas de sectores pobres y empobrecientes de resolver dicha tensión en procura de una vida digna. Pensar esta clave nos interpela acerca de actuales y futuras situaciones de vejez, de las consecuencias y a la vez perspectivas de transformación que dicha problemática tiene, para los viejos que son, para los viejos que seremos.

En nuestro encuentro cotidiano con los estudiantes, intentamos compartir la importancia de conocer desde la vivencia. Se trata de encontrar con ellos "en la realidad", algunas claves de comprensión, la que nos involucra y atraviesa; se trata de que no disocien formación académica con "vida personal"; intentamos que puedan verse, sentirse y pensarse como "uno", "entero". En relación a ello aparece muy fuerte la cuestión y reflexión ética; la discusión y toma de posicionamiento acerca de valores e idearios; de proyectos sociales y políticos; la dimensión política de la profesión. Consideramos que si bien la instancia de práctica académica no es el único dispositivo de enseñanza de la Intervención Profesional, de la enseñanza del oficio (ya que todos los espacios curriculares y disciplinas contribuyen a ello), sí constituye el escenario principal que los "conecta" con los espacios "reales", concretos, del ejercicio profesional; les brinda herramientas para pensar la construcción del espacio profesional; les posibilita identificar y analizar escenarios en relación a diferentes contextos en los cuales Trabajo Social se inserta e interviene; les permite analizar condiciones de trabajo y de inserción laboral; les posibilita ese tan ansiado encuentro "cara a cara" con el "otro" y "probar/se" en la generación de relaciones, vínculos; en sus intervenciones, que cuestiones "se bancan" y cuáles no; cómo avanzar y crecer; cómo no flaquear y aprender a sobrellevar y fortalecerse... Es muy difícil transferir "formas de", cada uno de ellos tiene que hacer su propia experiencia, su propio aprendizaje, enfrentar cuestiones más y menos gratificantes; hacer sus búsquedas; encontrar caminos, andarlos y desandarlos; "probar/se" si se trata de Trabajo Social lo que desean y les interesa, porque supuestamente es para toda la vida. Trabajar estos aspectos en forma individual y grupal, se torna un desafío para el equipo docente, trabajar representaciones, prejuicios, imaginarios, conceptos, desde los espacios seleccionados como Centros de Práctica.

Desde la postura explicitada, intentamos integrar en diferentes espacios y dispositivos: el trabajo docente, la extensión, la investigación y el posgrado. La continuidad y profundización en la temática posibilitó la construcción de líneas de trabajo y producción; nuestra necesaria capacitación y actualización ${ }^{3}$ y el desarrollo de investigaciones; el aprendizaje y trabajo interdisciplinario para lograr un proceso real de articulación entre las "funciones" mencionadas. Nuestra experiencia surge a partir de interrogantes provenientes de la práctica docente y profesional en la temática, los que se tornaron en objeto de extensión universitaria primero y de investigación después. El involucramiento de estudiantes en cada una de ellas, fue y es muy significativo, tanto por sus cuestionamientos y aportes, como por la posibilidad que les brinda la incorporación en los proyectos como alumnos, becarios algunos, colaboradores otros; contribuyendo a su formación y aprendizaje y a sus futuras prácticas profesionales. En más de 20 años, hemos venido indagando acerca de las perspectivas teóricas vigentes y categorías conceptuales que atraviesan el campo y que a la vez permiten conocer, comprender, "nombrar", aquellas situaciones, hechos, fenómenos, sobre los que Trabajo Social interviene con la intencionalidad de modificar, aportar a transformaciones, cuando estos se han vuelto problemáticos. Entre los aspectos ligados al proceso de
3) Graduación como Magíster en Trabajo Social FTS/UNER. Graduación como Especialistas en Gerontología,
2009, EGCl, Universidad Nacional de Mar del Plata y Ministerio de Desarrollo Social de Nación 
envejecimiento abordados, destacamos: cuestión social, protección social; seguridad social, políticas sociales, salud, familia, redes sociales e institucionales de apoyo, espacios socioeducativos y culturales, relaciones intergeneracionales, institucionalización y alternativas a la institucionalización, cuestiones del trato, cuidado domiciliario de ancianos.

Entre los principales espacios construidos, destacamos:

- Proyecto de Extensión "Llegar a viejo". Abordaje de la Temática

- Problemática de la Vejez - 1995/2010 - 2014/2016 - 2016/2018

(con dos publicaciones).

Objetivos generales: brindar herramientas que contribuyan a instalar en la agenda pública, la problemática social del envejecimiento en relación a la protección social, como una de las problemáticas sociales centrales en las próximas décadas; Generar perspectivas teórico-metodológicas en el campo que permitan un mejor abordaje de diferentes situaciones de vejez.

Líneas de trabajo: a) vejez, protección social y políticas sociales; b) gerontología social e institucional; c) espacios socioeducativoculturales desde la perspectiva de crítica de la vida cotidiana y construcción de ciudadanía.

- Proyecto de Investigación "Envejecimiento y vejez. Espacios socioeducativos-culturales en el proceso de envejecimiento de viejos/as de sectores de pobreza de la ciudad de Paraná. Un estudio desde Trabajo Social".

En el marco del Programa de la UNER, Nóveles Directores, 2008/2010. Publicación Envejecimiento y espacios grupales Editorial Espacio 2012.

- Creación de la Carrera de Especialización en Gerontología. Aprobada por CONEAU (Comisión Nacional de Evaluación y Acreditación universitaria - Ministerio de Educación de la Nación) (sin implementar aún).

- Proyecto de Investigación "Envejecimiento y vejez. Procesos de envejecimiento y Configuraciones familiares en el actual contexto", 2011/2013.

En el marco del Programa PID/UNER. En proceso de publicación.

Consideramos que nuestra Facultad, aun teniendo en cuenta la situación presupuestaria crítica del Sistema Universitario Nacional, propicia este tipo de experiencias, a través de la predisposición y permanente apoyo.

\section{A modo de cierre}

Tal como explicitáramos, tenemos la convicción de que pueden integrarse las funciones básicas de la universidad, y que ello mejora y fortalece la formación de los estudiantes. Para ello es necesario el apoyo y sostenimiento institucional a nivel Facultad y Universidad y requiere mayor asignación presupuestaria dado que la tarea docente de grado, la que para nosotras constituye la base de la propuesta, se torna mucho más compleja e implica mayor tiempo dedicado al estudio, actualización, investigación, gestión de recursos, coordinación, generación de espacios y dispositivos de encuentro y de discusión, elaboración de artículos, ponencias, informes, etc.

En nuestro caso, las cuestiones, temas, problemas; los conceptos, categorías, relacionadas a Trabajo Social como profesión, como disciplina en construcción y sus dimensiones constitutivas (carácter interventivo e investigativo), tienen que orientar y otorgarles sentido a la práctica docente, de producción de conocimiento y de extensión universitaria, de quienes nos desempeñamos en el ámbito de la universidad pública, en la formación de trabajadores sociales, máxime en los espacios curriculares específicos. En esta línea, la universidad tiene que afrontar una reflexión crítica permanente sobre su papel en la producción, reproducción y distribución de un valor social privilegiado como es el conocimiento, en el fortalecimiento del espacio público y el ejercicio de ciudadanía, en pos de la construcción de una sociedad más justa, fraterna, solidaria, democrática, igualitaria.

¡Ojalá podamos continuar comprometidos con nuestros idearios, desde la pasión y la razón indisolubles, puestas en el oficio y la tarea!

\section{Referencias bibliográficas}

AA. VV. (2003). Formación Académica en Trabajo Social. Una apuesta política para repensar la profesión. FTS-UNER. Buenos Aires: Espacio.

AA. VV. (2007). Documento sobre Extensión Universitaria. Proyectos de Extensión. FTS-UNER.

Almeida Pastor, M. (1993). Apuntes sobre extensión universitaria. Universidad, (6), diciembre. Revista de la Universidad Técnica del Norte, Ibarra, Ecuador.

Freire, P. (1997). Pedagogía de la Autonomía. Siglo XXI Editores.

Giroux, H. (1990). Los profesores como intelectuales: hacia una pedagogía crítica

del aprendizaje. Barcelona: Paidós.

lamamoto, M. (2003). El Servicio Social en la contemporaneidad. São Paulo:

Cortez. Primera Parte.

Lera, C. (2006). Ponencia en Encuentro Académico Nacional de FAUATS. UN de Luján.

Ludi, M.C. (2009). Diseño Carrera de Especialización en Gerontología. FTS-UNER. Programación Académica Anual. Equipo Docente Asignaturas Intervención Profesional y Vida Cotidiana ( $3^{\circ}$ año) e Intervención Profesional e Institucionalidad Social (4ำ año). Aprobada por Resolución CD Ciclo Lectivo 2012.

Matus, T. (1999). Propuestas Contemporáneas en Trabajo Social. Hacia una Intervención Polifónica. Buenos Aires: Espacio.

Rozas Pagaza, M. y Ludi, M.C. (2009). Algunas reflexiones generales sobre el Trabajo Social argentino. Servicio Social \& Sociedade, (100), noviembre. São Paulo: Cortez.

UNER. Informes finales (2007/2010/2014/2016). Proyecto de Extensión "Llegar a viejo". FTS-UNER.

UNER. Informes finales (2010/2012/2015). Proyecto de Investigación. FTS-UNER 